\title{
LA CONCEPTION DE SERIOUS GAMES OU JEUX VIDÉO ÉDUCATIFS : QUELLES CONVERGENCES DE MÉTIER ENTRE ENSEIGNANT ET GAME DESIGNER?
}

\author{
Mériem El Mansouri ${ }^{1}$, Nicole Biagioli ${ }^{2}$
}

Tout jeu à intention pédagogique implique que ses créateurs mobilisent des compétences d'ingénierie didactique. Nous postulons que la conception d'un jeu vidéo pour apprendre nécessite des compétences croisées d'enseignant et de game designer. Nous montrons qu'il existe au plan théorique une série d'analogies structurelles entre les modèles dégagés par la recherche sur le jeu vidéo et ceux dégagés par la recherche sur l'enseignement-apprentissage. Ces analogies nous permettent d'établir un modèle théorique du gameplay pour apprendre et de son opérationnalisation, jetant ainsi les bases d'une ingénierie de conception mixte. Il apparaît que les schèmes opérationnels de ces deux métiers sont homologues : leur capacité à gérer l'écart entre les prévisions et la réalité de l'action, grâce à des leviers qui leur sont propres, est à l'origine du croisement des compétences, nécessaire à la conception de jeux vidéo éducatifs.

1 Mériem El Mansouri est doctorante au CTEL (Centre Transdisciplinaire d'Épistémologie de la Littérature et des Arts vivants), Université Nice Côte d'Azur.

2 Nicole Biagioli est professeure émérite de langue et littérature françaises, CTEL, Université Nice Côte d'Azur.

Recherches en communication, $\mathrm{n}^{\circ} 49$ - Article publié le 21/03/2019 
L'acte de jouer et l'acte d'apprendre sont mis en corrélation dans le monde académique par plusieurs champs de recherche. Cette relation est aussi très présente dans les offres des professionnels du marché économique de l'éducation et de la formation. Depuis une quinzaine d'années, l'outil numérique mis en avant pour faire jouer et apprendre en même temps est le jeu vidéo éducatif, communément appelé jeu sérieux ou serious game en anglais.

En effet, le jeu vidéo éducatif apparaît comme le média qui permet d'associer le ludique et le didactique puisqu'il prétend offrir d'un côté la liberté et la gratuité propres au jeu et, de l'autre, les conditions nécessaires à l'apprentissage. Mais, la mise en œuvre de cette relation aussi bien dans la conception que dans l'utilisation apparaît plus complexe qu'on ne l'imagine de prime abord. Toute la difficulté est de mettre en place une ingénierie de conception capable d'associer harmonieusement les composantes ludiques et didactiques dans l'expérience de jeu proposée aux utilisateurs, puisque ce type de jeux ne s'arrête pas au seul divertissement mais vise aussi à faire apprendre des savoirs.

Depuis quelques années, des retours d'expériences et des articles de recherche commencent à pointer les défauts des jeux vidéo éducatifs disponibles sur le marché aussi bien au plan didactique qu' au plan ludique (Lavigne, 2015 ; El Mansouri, Biagioli, 2016). Pourtant, ce n'est que depuis peu que l'idée est venue de rassembler des représentants de la recherche, des studios de jeux vidéo, des entreprises de serious games, des enseignants et des game designers afin de les mettre en relation le temps d'une journée ou deux dans l'espoir qu'ils collaborent et créent des jeux vidéo éducatifs qui répondent à leur double mission ${ }^{3}$.

Néanmoins, pour y avoir participé, il nous est apparu que ces regroupements de communautés de praticiens et de théoriciens souffraient d'un manque de préparation en amont. Celles-ci, selon nous, devraient faire intervenir des chercheurs issus des études du jeu vidéo et de la didactique, deux champs qui nous semblent

3 Comme vient de le faire le dernier séminaire Gamesforchange sur l'éducation et le jeu vidéo à Paris les 19 et 20 novembre 2018. 
majeurs, mais qui sont peu représentés voire inexistants dans les salons et séminaires professionnels ou mixtes ${ }^{4}$.

Une réflexion préalable sur la conception et le développement de ces jeux pourrait créer des ponts entre les différents acteurs concernés par cette ingénierie didactique spécifique. La recherche, en modélisant les pratiques, pourrait permettre de faire le lien entre les métiers d'enseignant et de game designer. Nous postulons en effet que la conception d'un jeu vidéo éducatif nécessite des compétences croisées d'enseignant et de game designer qui peuvent être apportées par des professionnels différents comme coexister chez le même individu.

Dans cet article, nous montrons qu'il existe au plan théorique une série d'analogies structurelles entre les modèles dégagés par la recherche sur le jeu vidéo et ceux dégagés par la recherche sur l'enseignement-apprentissage. Celles-ci permettent de poser les bases d'une collaboration entre game designer(s) et enseignant(s) dans la conception des jeux sérieux.

Pour ce faire, après avoir explicité les concepts fondamentaux de la didactique nécessaires au développement de notre réflexion, nous proposerons une définition du jeu sérieux apte à fusionner les registres ludique et didactique dont il relève génériquement. Ensuite, notre propos se centrera sur la dimension transdisciplinaire d'une approche didactique de l'ingénierie de conception. Nous présenterons deux modèles théoriques à partir desquels nous établirons les correspondances et les complémentarités entre les compétences professionnelles du game designer et celles de l'enseignant.

\section{Un cadre théorique interdisciplinaire}

\subsection{Approche didactique}

Le terme « jeu » peut prêter à confusion dans la langue française par rapport à la langue anglaise. Il désigne aussi bien le jeugame, l'artéfact employé pour jouer et ses règles d'emploi, que

4 C'est-à-dire des événements qui admettent des chercheurs à côté des professionnels. 
le jeu-play, c'est-à-dire la situation construite avec le jeu-game, autrement dit l'activité ludique ou le playing. L'équipe du Jen.lab s'appuie sur les travaux d'Henriot (1989) pour considérer qu' « un jeu-game n'est qu'une proposition. C'est l'attitude ludique du joueur, le playing, qui signe la mise en place du jeu-play » (Loup et al., 2015). Dans cette étude, nous n'allons pas penser le jeu uniquement en tant qu'artéfact, mais plutôt prendre en compte les interactions qui émergent de la situation : le jeu-play.

Tout comme Loquet (2009), nous dirons que le jeu est d'abord une activité, avec deux dimensions principales :

- La dimension «affective » : le jeu est une activité spontanée qui exige un état de détente sur le plan psychique, d'où son caractère récréatif. Dès qu'une activité ludique devient utilitaire et possède un but extérieur, qu'il soit pédagogique, informatif ou communicationnel, elle perd l'attrait et les caractéristiques du jeu gratuit.

- La dimension " effective » : le jeu renvoie à une vision plus organisée et pragmatique d'une activité, avec un système de règles, explicites et spécifiques, qui définit précisément un succès et un échec, un gain et une perte. Il suppose donc que les comportements mus par le plaisir, loin d'être aléatoires, sont conditionnés par un ensemble de règles (Loquet, 2009, p. 92).

Cette dernière dimension explique pourquoi des didacticiens ont modélisé l'apprentissage sous forme d'un jeu. La théorie de l'action conjointe en didactique (TACD) (Sensevy \& Mercier, 2007) repose sur la mise en relation des jeux d'apprentissage avec les jeux épistémiques ${ }^{5}$. Cette modélisation permet de com-

5 Un jeu épistémique d'épistémè (grec, savoir) est un jeu qui permet d'acquérir et/ou de transmettre des savoirs. Il y a plusieurs types de jeux épistémiques : le jeu (ou capacité) épistémique émergent, le jeu épistémique source, le jeu (ou capacité) épistémique cible. Les jeux épistémiques émergents sont les capacités effectives que l'apprenant a acquises pendant les jeux d'apprentissage. Le jeu épistémique source représente les usages de l'expert; le jeu épistémique cible correspond à la transposition didactique, c'est-à-dire « l'art » d'introduire les usages des experts dans la situation d'apprentissage, autrement dit la capacité de transposition que l'enseignant met en œuvre afin d'approcher la pratique de l'expert (G. Sensevy, 2011, pp. 123-131). 
prendre de façon théorique comment le jeu-play didactique peut être associé à une situation didactique composée d'un système enseignant-élève-milieu. Nous pouvons généraliser la situation de classe à toutes les situations d'apprentissage, pour peu qu'il y ait un adulte dans la posture de l'enseignant et un enfant ou des enfants dans la posture de l'apprenant.

Une activité didactique peut prendre la forme d'une activité ludique (en utilisant d'ingénieuses transactions et stratégies d'apprentissage), mais doit garder la dimension irréductible de l'apprentissage : elle doit faire prendre conscience des savoirs qu'elle véhicule, les faire assimiler et approfondir.

Les game designers Crawford (2003) et Koster (2013) s'accordent à dire que, dans le jeu vidéo, jouer en ayant du plaisir revient à apprendre. On retrouve les deux dimensions affective et effective du jeu en tant qu'activité aussi bien dans la progression ludique du jeu vidéo que dans la progression didactique du jeu vidéo éducatif. Cependant, la différence se fait sur les objectifs et leurs primautés. L'objectif premier du jeu vidéo est le divertissement ; il est fondé sur l'apprentissage par le joueur des savoirs et des compétences de jeu. L'objectif premier du jeu vidéo éducatif est didactique ; il est fondé sur l'apprentissage par l'apprenant des savoirs et des compétences du domaine éducatif visé.

Dans sa théorie des situations didactiques, Brousseau (1998) définit une situation a-didactique comme la part de la situation didactique $^{6}$ dans laquelle l'intention d'enseigner n'est pas explicite au regard de l'apprenant. Cette théorie nous permet d'identifier le type de situation didactique à atteindre avec le jeu vidéo éducatif. L'objectif qualitatif de la création d'un jeu sérieux est atteint si la mise en œuvre ainsi que les caractéristiques de son jeu-game permettent d'atteindre cette situation a-didactique. Or du même coup, l'assimilation du jeu sérieux à une situation a-didactique pointe sa lacune didactique constitutive qui est sa difficulté à faire prendre conscience des savoirs qu'il véhicule, à les faire

6 Une situation est didactique lorsqu'un individu a l'intention d'enseigner à un autre individu un savoir donné. 
assimiler et approfondir, en raison de son potentiel de diversion, ou de divertissement.

Le milieu didactique est le système de ressources données par la situation, qui permet et oriente l'action des apprenants et de l'enseignant (Brousseau, 1990). Lorsque le milieu est a-didactique, l'apprentissage est conçu comme l'adaptation de l'apprenant au milieu. Il est organisé de telle façon que les relations établies par l'apprenant avec le milieu procèdent des rétroactions qu'il en reçoit et non des exigences ou des intentions didactiques du professeur. En d'autres termes, l'apprenant joue avec le milieu. L'enseignant se borne alors à des interventions neutres par rapport aux savoirs en construction et se contente d'encourager et de constater les faits, sans exigence particulière.

Le jeu sérieux en tant qu'artéfact (jeu-game) correspond donc à ce milieu didactique spécifique que Brousseau appelle le milieu a-didactique, et qui permet à l'élève d'apprendre en jouant et en étant autonome. À noter que, d'ordinaire, c'est l'enseignant qui conçoit le milieu a-didactique et qui prépare en amont l'activité d'apprentissage, fixe les objectifs didactiques et les prérequis sous forme de compétences et de connaissances.

Rappelons ici en quoi le savoir se différencie de la connaissance. La connaissance est une construction personnelle de l'apprenant qui lui est propre et renvoie à une intériorisation du savoir. Dans le milieu a-didactique, que celui-ci soit constitué par un jeugame ou tout autre dispositif, cette construction se fait en réagissant avec le milieu.

Toute situation a-didactique débute par un processus de dévolution : l'enseignant fait en sorte que les apprenants assument la responsabilité de l'apprentissage. Elle se clôt par une phase d'institutionnalisation : les apprenants avec l'aide du professeur redécontextualisent, redépersonnalisent la connaissance qu'ils ont produite. Le but est de reconnaître dans ce qu'ils ont fait quelque chose qui a un caractère universel, un savoir culturel réutilisable. C'est ainsi qu'ils transforment leurs réponses et leurs connaissances en savoirs.

La dévolution et l'institutionnalisation sont des processus liés à l'articulation savoirs-connaissances, des phases nécessaires à l'ap- 
prentissage et organisées par l'enseignant. Le rôle de l'enseignant qui est régulateur pendant la situation a-didactique devient primordial lors des processus de dévolution et d'institutionnalisation pendant lesquels l'enseignant doit donner du sens aux connaissances. Dans le cas de l'utilisation du jeu sérieux en totale autonomie, ce sont les liens « aide», le tutoriel, un livret d'accompagnement et/ou la mise en place d'une narration adaptée qui permettent à ces deux phases d'exister dans la situation d'autodidaxie?

En définitive, l'approche didactique modélise le jeu en tant que milieu. L'apprentissage a lieu parce que l'activité de jeu est encadrée par des phases de dévolution et d'institutionnalisation. Lorsque le jeu est utilisé en classe, le rôle de l'enseignant est prépondérant avant et après la phase dite a-didactique. Lorsque le contexte d'utilisation n'est pas scolaire, cela présuppose que le concepteur du jeu puisse inclure des éléments permettant cet encadrement hors de la présence de l'enseignant. De plus, l'approche didactique insiste sur la nécessité d'intérioriser le projet didactique dans le jeu car il est facteur d'apprentissage en lui-même : quand on joue, on apprend à jouer. Si le jeu est utilisé seul, il faut y intégrer les phases d'institutionnalisation et de dévolution, sans que cette intégration lui fasse perdre sa capacité à être identifié en tant que jeu.

Pour la suite, nous allons compléter notre propos en précisant ce que nous définissons en tant que jeu vidéo à visée éducative dans le cadre d'une approche mixte issue des champs des game studies et de la didactique pour présenter ensuite les spécificités de l'ingénierie de conception correspondante.

\subsection{Le serious game, un jeu vidéo éducatif?}

Selon le domaine dans lequel nous plaçons la réflexion, le serious game ne se définit pas de la même manière. Dans le registre didactique, on définit le serious game en tant que jeugame, milieu a-didactique dans le « jeu-play» didactique. Dans

7 L'autodidaxie est l'acquisition de connaissances par un individu en dehors des dispositifs éducatifs officiels (établissements scolaires, centres d'apprentissage ou de formation) et sans intervention d'un enseignant ou d'un formateur. 
le registre sociotechnique, nous retenons la définition fondatrice d'Alvarez :

Application informatique, dont l'objectif est de combiner à la fois des aspects sérieux (serious) tels, de manière non exhaustive, l'enseignement, l'apprentissage, la communication, ou encore l'information, avec des ressorts ludiques issus du jeu vidéo (game). Une telle association a donc pour but de s'écarter du simple divertissement. (Alvarez, 2007, p. 3)

Même si, par commodité, nous assimilons « serious game » et « jeu vidéo éducatif », nous avons souhaité différencier les deux registres en associant « jeu vidéo éducatif » au cadre didactique et «serious game» au cadre sociotechnique.

En effet, le cadre pluridisciplinaire de cette étude exige que nous prenions en compte le concept d'attitude ludique défini par Henriot et repris par Genvo. Genvo précise que concevoir un jeu c'est : « [...] communiquer par une structure l'idée de jeu à autrui afin de lui faire adopter cette attitude » (Genvo, 2008, p. 1); dans la mesure où :

[...] une structure conçue à des fins de jeu, pour être reconnue comme jouable (et actualisée ensuite sur le mode de l'attitude ludique), va puiser au sein de la culture dans laquelle elle s'inscrit certaines représentations du jeu qui permettent de partager cette signification. (Genvo, 2008, p. 4)

C'est pourquoi l'auteur préfère parler de play design plutôt que de game design. Pour concevoir un jeu vidéo, le game designer doit construire une structure de jeu caractérisée par une jouabilité et un éthos ludique ${ }^{8}$ capable de communiquer une attitude ludique à différents types de joueurs en fonction de leurs compétences et connaissances ludiques.

Ceci nous a conduit à proposer la définition suivante du serious game:

8 L'éthos ludique correspond aux « valeurs conférées à l'activité ludique par un logiciel pour être actualisé comme jeu » (Genvo, 2012). 
Une application informatique que la majorité des joueursapprenants doit pouvoir identifier en tant que jeu vidéo mais dont l'intention première est d'enseigner des savoirs. L'expérience ludo-éducative proposée par cet artefact doit résulter d'une progression issue d'un scénario ludique et d'un scénario didactique intimement liés. (El Mansouri, 2017b)

Ce faisant, nous souhaitons différencier notre approche de la conception de celles existantes. En effet, lors d'une veille ${ }^{9}$ que nous avons effectuée sur les jeux actuellement disponibles, nous avons rencontré trois types d'approches que nous allons illustrer chacune par un exemple. Afin d'expliciter notre propos, nous schématisons chaque démarche sous forme d'une progression temporelle de boucles de gameplay et/ou de boucles d'apprentissage selon le cas, qui correspond à une progression de séquences soit de jeu, soit d'apprentissage, soit des deux :

- La première est celle d'un scénario ludique prépondérant sans progression didactique associée dans lequel seuls quelques éléments de savoirs sont dispersés. Par exemple, les concepteurs remplacent un objet de collecte classique dans un jeu de plateforme (ex. : les pièces dans le jeu Mario) par des hamburgers ou des fruits dans un jeu sur la nutrition comme Nutribird (jeu analysé par El Mansouri et Biagioli, 2016).

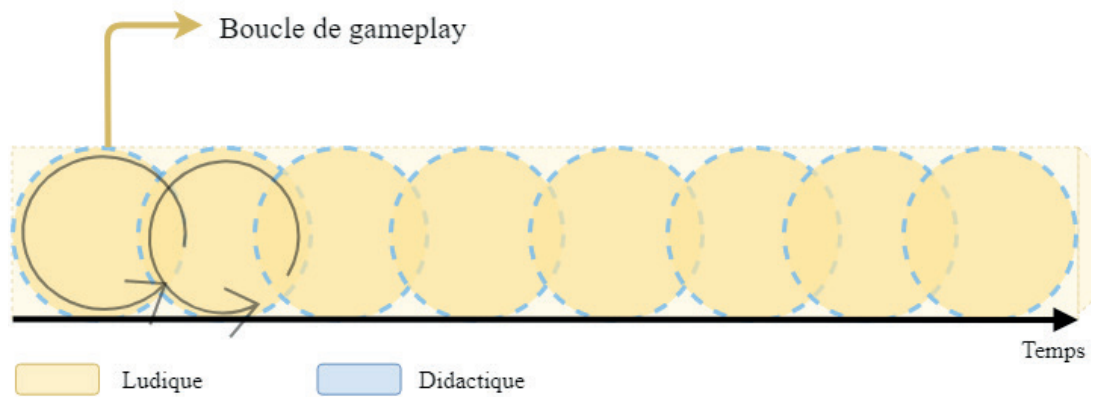

Figure 1. Illustration schématique de la première approche

9 Veille web de serious game récents (depuis 2015) pour enfants à visée pédagogique qui nous a permis de recenser à ce jour une trentaine de jeux. 
- La deuxième est celle d'un scénario didactique prépondérant qui se base souvent sur des exercices de type scolaire ou des quiz et les présente en tant que jeux en tablant sur leurs caractéristiques numériques et interactives pour apporter le côté ludique comme Méli-mélo, (jeu analysé par El Mansouri et Biagioli, 2016);

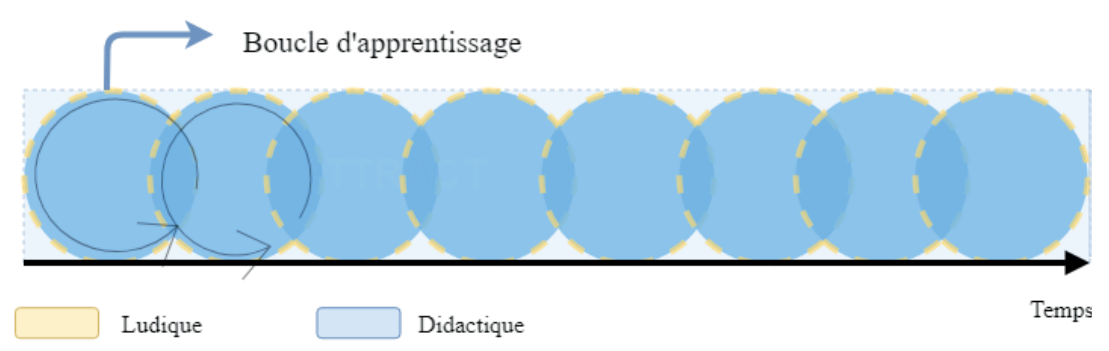

Figure 2. Illustration schématique de la deuxième approche

- La troisième croise un scénario ludique avec des exercices (ou quiz) interactifs en temps limité selon une progression alternée (ex. : Navadra: Math \& Magic $^{10}$ ). Il faut chaque fois réussir l'exercice pour être admis à jouer.

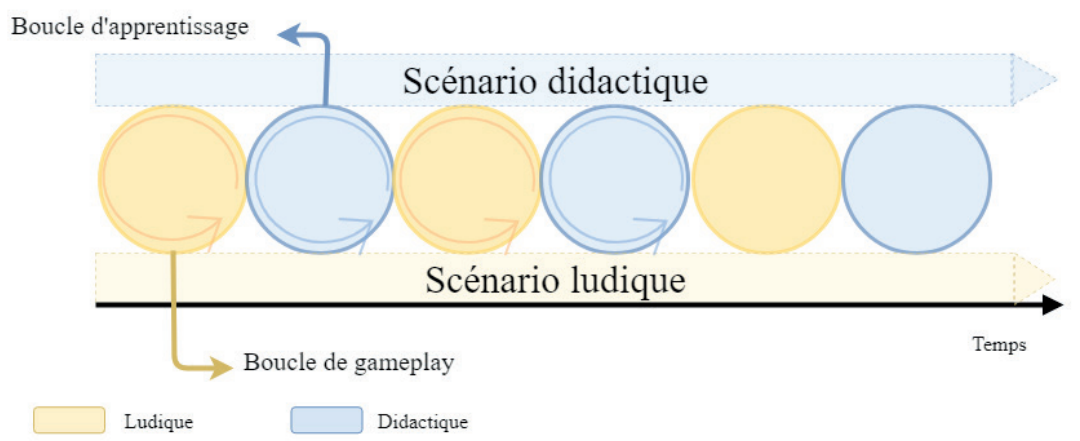

Figure 3. Illustration schématique de la troisième approche

Dans notre approche, nous proposons de fusionner d'emblée les deux scénarios en les alignant sur une même progression.

$10 \mathrm{https} / / /$ www.navadra.com/collegiens/ 


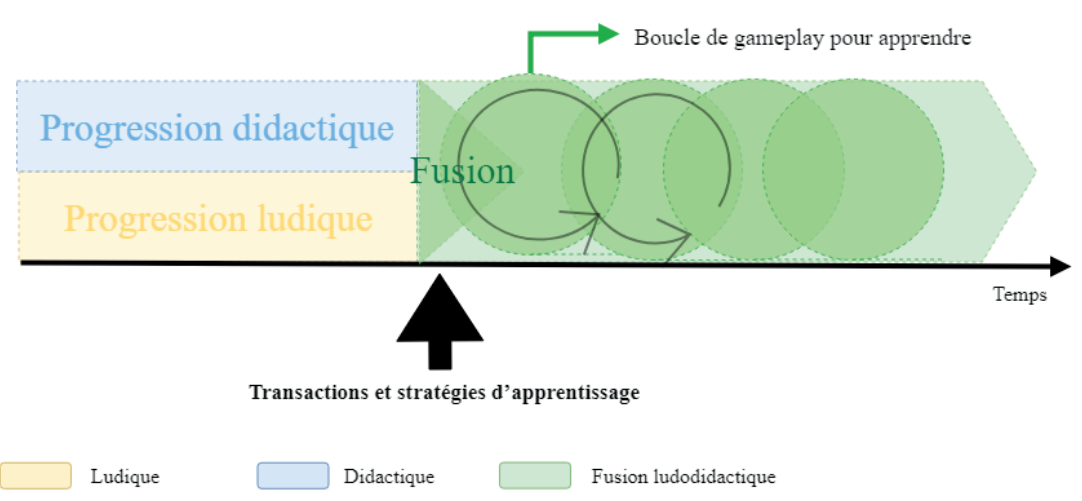

Figure 4. Illustration schématique de la fusion des progressions

D'une part, le jeu doit rester un jeu et ne doit pas être perçu en tant qu'exercice scolaire, d'autre part le jeu doit rester vecteur d'apprentissage sans se limiter à sa dimension ludique. Notre approche permet de garder les caractéristiques intrinsèques du jeu grâce à la fusion des objectifs ludiques et didactiques dans une boucle de gameplay pour apprendre.

Dans le cadre ludique, Genvo a théorisé la boucle de gameplay avec un modèle sémiotique du gameplay. Afin de faire le lien avec le cadre didactique, nous intégrerons par transposition de concepts des composantes didactiques pour construire un modèle sémiotique du gameplay pour apprendre.

\section{Une ingénierie de conception mixte}

\subsection{Modèle sémiotique du gameplay pour apprendre}

Genvo définit le gameplay comme la dynamique d'appréhension des règles par l'action qui permet de mettre au centre de la réflexion les compétences qu'un acteur doit acquérir pour réaliser un objectif (Genvo, 2013). Il établit la modélisation du gameplay à partir d'un cadre théorique mixte issu de la ludologie et de la grammaire narrative greimassienne. Pour décrire la progression narrative, Greimas s'appuie sur les valeurs modales signifiées par les verbes devoir, pouvoir, savoir et vouloir (Grei- 
mas, 1970, p. 179-181). Celles-ci correspondent aux relations qui structurent le schéma actanciel (Ibid., p. 268). Genvo a transposé le modèle narratif de Greimas pour construire un modèle sémiotique du gameplay en distinguant le game (artéfact) du play (attitude ludique) d'après les travaux d'Henriot (1989).

Selon Genvo, tout l'art du game design consiste à donner envie de surmonter les obstacles pour réaliser le projet ludique. De même, nous pouvons dire que l'art d'enseigner consiste à donner envie de surmonter les obstacles pour réaliser le projet didactique.

Pour Genvo, le joueur, en s'appuyant sur ses compétences et sa culture ludique, passe par une série de conflits et relève des défis pour réaliser le projet ludique. De son côté, un enseignant peut construire son projet didactique sur une stratégie basée sur la démarche de résolution de problème. L'apprenant, en s'appuyant sur ses compétences et ses prérequis, trouve des solutions en passant par une série de conflits cognitifs (Perraudeau, 2006) qui l'obligent à revenir sur ses hypothèses pour à terme relever les défis didactiques.

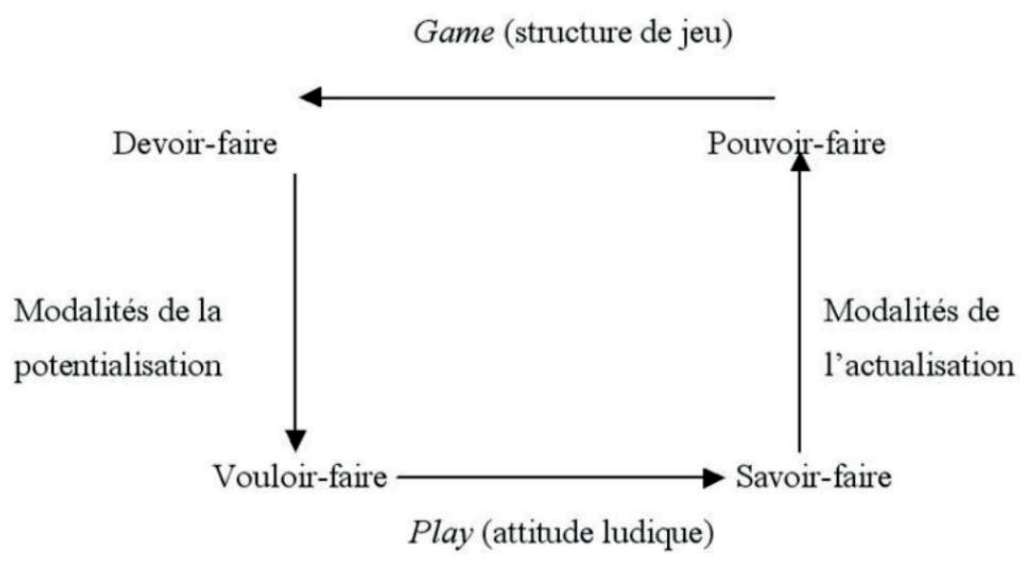

Figure 5. Modèle sémiotique du gameplay, Genvo (2008, p. 11)

Le devoir-faire et le pouvoir-faire dépendent tous les deux du game (structure du jeu). L'un correspond aux objectifs à atteindre et l'autre aux moyens pour progresser dans l'intrigue. Dans le jeu vidéo, il est possible d'expérimenter des pouvoir-faire que l'on n'a 
pas à disposition tous les jours : utiliser des armes de plus en plus performantes et diversifiées, rouler dans des voitures de sport à toute vitesse, se servir de personnages avec des caractéristiques de combat différentes, etc.

Le vouloir-faire et le savoir-faire dépendent tous les deux du play (attitude ludique). L'un correspond à la motivation à jouer du joueur et l'autre à la courbe d'apprentissage du jeu : progression des savoir-faire (skills) pour pouvoir explorer les pouvoir-faire du jeu (ex. : arbre de compétences dans les jeux de rôle).

Nous identifions 4 correspondances avec la didactique :

- Pouvoir-faire : prérequis (connaissances et/ou compétences préalablement acquises nécessaires à l'activité) ;

- Devoir-faire : objectifs pédagogiques/didactiques ;

- Vouloir-faire : motivation et appropriation (ex. : donner du sens aux apprentissages) ;

- Savoir-faire : compétences à consolider ou à développer (ex. : construire une stratégie de résolution de problème).

Le schéma suivant adapte donc le modèle de Genvo aux jeux vidéo éducatifs, en intégrant les composantes didactiques :

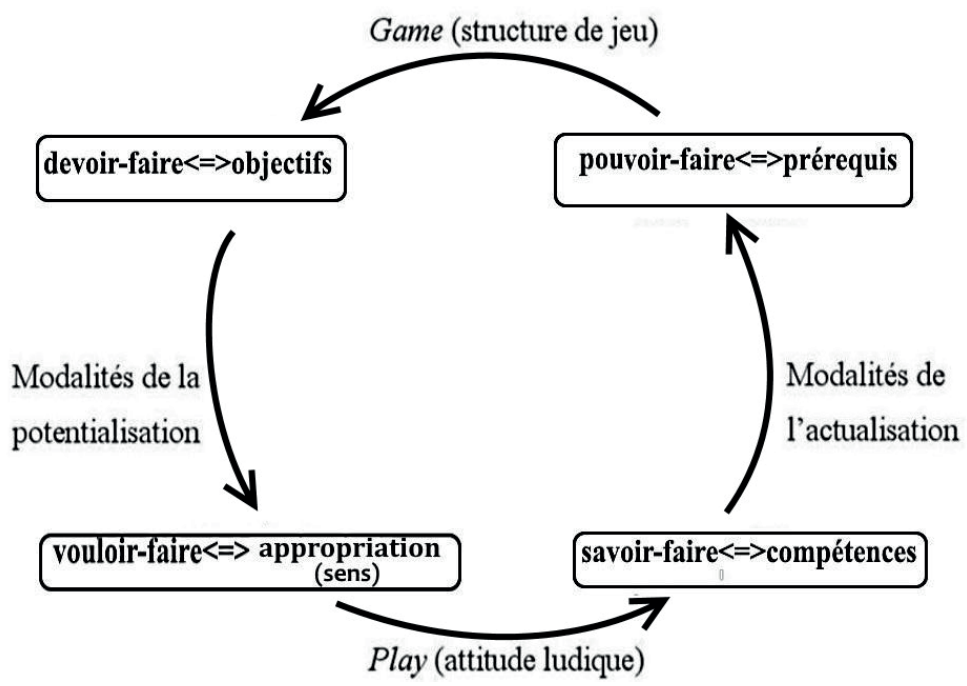

Figure 6. Modèle sémiotique du gameplay pour apprendre (El Mansouri, 2017a) 
Maintenant que nous disposons d'un modèle qui permet de mettre en corrélation les différents éléments d'une boucle d'apprentissage et les différents éléments d'une boucle de gameplay, nous allons identifier les analogies entre les conditions d'une expérience vidéoludique réussie et celles d'une expérience didactique efficace.

\subsection{Fun et flow}

Le psychologue Csíkszentmihályi (2008) définit le flow (flux) comme un sentiment de concentration totale provoqué par une activité qui maintient un haut niveau de plaisir et de satisfaction. Plongés dans leur activité, les joueurs en oublient le temps qui passe. Dans le jeu vidéo, on parle de zone de flow (Chen, 2006) dans laquelle l'expérience de flow doit être maintenue en respectant l'équilibre fondamental entre la difficulté des défis à relever et les compétences du joueur.

On peut aussi imaginer que dans une situation a-didactique, en prenant la responsabilité de la tâche et en relevant les défis didactiques, l'apprenant, à l'instar du joueur, subit une forte distorsion de la perception du temps. Il est maintenu dans cet état de concentration de la même manière que le joueur est maintenu en zone de flow.

Cependant, le flow, tant ludique qu'a-didactique, n'est pas un état stable et permanent, mais plutôt, comme le dit Koster (2013), une série de petits pics de plaisir. En effet, il est en pratique très difficile de l'atteindre, car il dépend de la qualité de l'expérience proposée par le jeu, de son contexte d'utilisation, du joueur luimême avec sa culture vidéoludique et ses compétences propres.

C'est pourquoi Koster fait une place à côté du flow à un autre état gratifiant, le fun, plus facile à atteindre (Koster, 2013, p. 96-98). Il définit le fun comme une sensation de bien-être proche de celle provoquée par les drogues. Il parle d'apogée du fun lorsque le joueur arrive à comprendre une tâche et qu'il résout un problème. L'absence de flow n'exclut pas le fun. Koster dit même que certains flows peuvent ne pas être amusants : c'est le cas de nombreuses activités, comme la méditation. 
Koster fait même le rapprochement avec la « zone de développement proximal $\rangle^{11}$ en éducation, en rappelant que l'apprenant a des tâches à accomplir, certaines qu'il sait faire, d'autres pas, d'autres encore qu'il ne peut réaliser sans aide. Dans ce dernier cas, le fun est provoqué par l'aide que fournit le système de jeu. Tous les cas de flow se rapportent quant à eux à des situations de totale maîtrise.

Nous considérons donc la maîtrise de l'activité ( flow) comme l'objectif vers lequel nous voulons tendre par le biais de l'apprentissage avec le jeu (fun). Une fois atteint, cet objectif provoque l'absorption cognitive qui « [...] fait que les individus qui l'ont connue une fois n'ont de cesse de vouloir réunir les conditions susceptibles de la provoquer à nouveau » (Fenouillet, 2014), ce qui installe le mécanisme de l'autodidaxie.

L'approche didactique nous a permis de décrire théoriquement l'activité du joueur seul devant un écran comme une succession de phases a-didactiques, encadrées chacune par une phase de dévolution et une phase d'institutionnalisation. Du coup, nous avons pu intégrer la dimension a-didactique dès la conception du gameplay. Nous avons établi un modèle du gameplay pour apprendre dont la mise en application permet tous types d'apprentissages fondés sur une stratégie de résolution de problème. Pour rendre ce modèle opérationnel, il faut, comme pour tout jeu, essayer de faire atteindre au joueur apprenant la zone de flow le plus vite possible et de l'y maintenir le plus longtemps possible. Dans le paragraphe suivant, nous allons voir en quoi cette opérationnalisation qui dépend des compétences et des stratégies du concepteur de jeu sérieux suit une série de règles que l'on retrouve aussi bien dans le ludique que dans le didactique. Nous allons en effet montrer les analogies entre les principes de régulation du flow énoncés par Chen et ceux énoncés par Brousseau.

11 La zone proximale de développement désigne « le niveau qu'atteint l'enfant lorsqu'il résout des problèmes non plus tout seul mais en collaboration ». Elle s'oppose au « niveau présent de développement » (ou âge mental), « qui est déterminé à l'aide des problèmes résolus de manière autonome » par l'enfant (Vygotski, 1997, p. 351). 


\subsection{Opérationnalisation du modèle}

En s'appuyant sur les travaux de Csíkszentmihályi, Chen (2006) établit trois éléments déterminants pour atteindre la zone de flow :

- Dans un premier temps, le jeu doit être directement gratifiant. Le joueur doit être motivé dès le départ, l'entrée dans le jeu doit être facilitée (phase d'appropriation, paragraphe 2.3.1);

- Dans un deuxième temps, la difficulté des défis à relever doit être adaptée aux compétences du joueur, ce qui lui permet de se plonger dans l'activité (équilibre de la progression dynamique, paragraphe 2.3.2);

- Dans un troisième temps, le joueur parvient à avoir un sentiment de contrôle, à se sentir maître de son activité (maîtrise de la progression, paragraphe 2.3.2).

Ces deux derniers points caractérisent la phase de progression. Si les trois éléments sont respectés, le joueur aura une perception modifiée du temps pendant son activité de jeu.

\subsubsection{L'appropriation}

La sensation d'immersion proposée par le jeu vidéo est un outil de connexion entre l'apprenant et le savoir. Elle débute par l'appropriation du jeu, aussi bien sur le plan ludique que didactique, qui elle-même dépend du sens que le joueur-apprenant donne à la mise en scène des savoirs et à la mise en scène ludique. Nous pensons que le déclenchement de cette prise de sens dépend de la qualité de l'immersion fictionnelle au sens que lui donne Schaeffer (1999, p. 148) : celui de feintise ludique partagée qui repose sur l'efficacité du semblant (Ibid., p. 188) procurée par les stimuli issus de la narration et de l'univers de jeu.

$\mathrm{Au}$ plan didactique, la motivation et l'entrée dans le projet didactique dépendra de la facilité avec laquelle l'apprenant prendra la responsabilité de relever les défis didactiques proposés par le milieu. Le milieu didactique doit être adapté à l'âge, aux com- 
pétences de l'élève et nécessite certains prérequis indispensables à l'appropriation. La qualité du milieu a-didactique proposé (jeugame) et la qualité de l'accompagnement sous la forme de la présence d'un adulte ou d'un livret pédagogique facilitera cette entrée dans l'activité didactique au même titre que l'univers de jeu et de sa narration.

\subsubsection{La progression}

La dynamique du gameplay et la dynamique du projet didactique progressent le long d'une ligne temporelle. Cette progression doit être porteuse de sens et sa conduite repose sur le respect d'équilibres d'ajustement spécifiques. Pour le jeu vidéo, Chen (2006) propose une régulation sous la forme de ce qu'il appelle le Dynamic Difficulty Adjustment ${ }^{12}$ (DDA), qui consiste en trois principes :

- Pour s'adapter aux différents profils de joueurs, c'està-dire couvrir une zone de flow plus large, un jeu doit élargir sa gamme d'expériences. Il doit proposer différents niveaux de lecture, du plus simple au plus complexe. Le joueur doit pouvoir identifier d'emblée le gameplay qui correspond à ses compétences afin de s'y plonger directement sans se perdre;

- Un jeu conçu pour une large audience doit aussi adapter son gameplay en fonction du joueur, ce qui place les compétences de jeu au centre de la conception. Par exemple, le créateur prévoit une régulation du niveau des ennemis dans les combats en fonction de la progression statistique du joueur dans le jeu, ce qui lui permet d'avoir des ennemis ni trop forts ni trop faibles. De cette façon, le joueur peut progresser à son rythme ;

- Un jeu bien conçu ne se contente pas de proposer une expérience de jeu linéaire. Par exemple pour relever un défi : il donne au joueur un choix de stratégies qui pourront avoir ou non des effets sur la suite narrative.

12 En français : ajustement dynamique de la difficulté. 
Chen conclut que le jeu doit être le plus dynamique et flexible possible pour permettre à chaque joueur d'entrer, de rester dans la zone de flow et de terminer le jeu.

Pour la didactique, Brousseau explique que la réussite de la conduite d'une situation a-didactique nécessite la gestion d'équilibres fondamentaux qui repose sur les choix didactiques et les qualités pédagogiques et psychologiques de l'enseignant : « La gestion de ces équilibres exige de nombreuses qualités pédagogiques et psychologiques mais elle repose d'abord sur des choix didactiques $»$ (Brousseau, 1990, p. 330).

Brousseau distingue ainsi six équilibres :

1. L'« équilibre (oscillations autour d'une position) entre incertitude et certitude, désordre et ordre, difficulté et facilité... » (ibid.).

Cet équilibre fait écho à l'équilibre entre les niveaux de difficultés préconisé par Chen (cf. supra), lorsqu'il décrit le deuxième élément déterminant de la zone de flow (équilibre de la progression dynamique).

2. L'« équilibre entre les niveaux de contrôles : l'esprit ne peut pas manier en même temps trop de conditions trop incertaines. La maîtrise de l'incertitude passe par une bonne répartition des charges entre les différents niveaux d'adaptation. Suffisamment de savoir bien connu, un peu de savoir en voie d'acquisition, une activité cognitive publique et privée juste suffisante pour justifier mais permettre aussi les interactions etc. » (Ibid.).

La maîtrise de l'incertitude évoque le sentiment de contrôle qui correspond au troisième élément déterminant de la zone de flow décrit par Chen (maîtrise de la progression).

3. L'« équilibre temporel et [le] rythme : si les savoirs et les algorithmes ne viennent pas assez vite soulager les modèles implicites et les connaissances, par conversion, par information ou par enseignement, la recherche personnelle s'essouffle (se complexifie, se sclérose et échoue), le contrat didactique se vide de son objet. Si au contraire ils viennent trop vite, la compréhen- 
sion peut n'avoir pas eu le temps de donner du sens à ces savoirs etc. » (Ibid.).

Nous pouvons rapprocher l'équilibre temporel et le rythme de Brousseau, de l'équilibre dont parle Chen que l'on doit maintenir pour rester en zone de flow et qui résulte de la mise en œuvre des trois principes du DDA.

4. L'« équilibre entre le plaisir de se définir par son activité intellectuelle et celui d'obtenir une sécurité reconnue de façon rapide et efficace, sans activité intellectuelle excessive par l'usage d'un savoir reçu » (Ibid.).

5. L'« équilibre entre le désir consommé (par les tâches difficiles ou peu gratifiantes ou simplement par l'accomplissement d'un projet...) et le désir produit (par des succès, des épreuves surmontées...)» (Ibid.).

Nous pouvons rapprocher ces deux équilibres des principes 2 et 3 de la DDA de Chen. Le niveau de compétences dont dépendent l'expérience et le plaisir du jeu doit être au centre du gameplay (adaptation des tâches). Le jeu ne doit pas être linéaire. Il doit permettre de contourner une difficulté ou d'utiliser une stratégie différente en cas d'échecs successifs ou de se lancer un défi plus difficile pour éviter ennui et frustration.

6. Enfin les « équilibres sociaux et culturels dans la classe entre le nombre de producteurs et de consommateurs d'idées, de réussites et d'échecs... » (Ibid.)

Lorsque Chen parle de profil de joueur et de la possibilité offerte au joueur d'identifier facilement son niveau par rapport au gameplay proposé (principe 1), cela correspond à un équilibre social et culturel, non pas dans une classe, mais au sein d'une communauté de joueurs.

Il apparait donc que les critères de gestion de l'expérience vidéoludique et de l'expérience a-didactique sont a priori analogues. Mais dans le cadre du jeu vidéo éducatif, ces principes sont confondus ou, comme nous le disions supra, fusionnés. La diffé- 
rence réside dans le réglage de ces principes grâce à des leviers propres à chacun des deux métiers enseignant et game designer. L'ingénierie de conception de jeux vidéo éducatifs repose sur la capacité du concepteur à faire usage des principes de régulation ludique et didactique et de leur complémentarité, donc de ces compétences croisées d'enseignant et de game designer.

\section{Conclusion}

Le modèle théorique du gameplay pour apprendre et l'optimisation de ses aspects qualitatifs permettent d'établir plusieurs correspondances et complémentarités entre les compétences professionnelles d'un enseignant et celles du game designer en tant que concepteur de jeu vidéo éducatif. Le défi pour les concepteurs du jeu-game est de proposer une structure de jeu capable de faire adopter une attitude ludique, mais aussi de faire apprendre. La qualité du serious game dépend de leur capacité à construire et réguler l'expérience qui sera proposée par le jeu dans ses deux phases d'appropriation et de progression.

La phase d'appropriation se décompose en tâches différentes : pour le projet ludique, ce sont des tâches majoritairement artistiques (graphisme, musique, narration, ergonomie, tutoriels, règles d'interaction) ; pour le projet didactique, des tâches majoritairement pédagogiques (prérequis, adaptation à l'âge et aux compétences). Dans les deux cas, ces tâches demandent des compétences psychologiques et pédagogiques pour sélectionner les éléments adaptés au public visé. Dans la phase de progression, la gestion des rythmes et des équilibres fait appel aux mêmes types de tâches et aux mêmes types de compétences, sauf que, pour le game designer, cette régulation se fera sur des leviers essentiellement techniques (feedback (animations, sons), interactions humain-machine, narration, etc.), alors que, pour l'enseignant, cela se fera sur des leviers didactiques et pédagogiques (exercices supplémentaires, rappels de leçon, accompagnement individuel ou travail de groupe).

Sur le plan théorique, nous avons montré que les schèmes opérationnels de ces deux métiers sont homologues et que la conception de 
jeux vidéo éducatifs repose sur le croisement de leurs compétences. C'est par la formation professionnelle, le partage d'expérience et la coopération entre les deux métiers que l'on pourra envisager une véritable hybridation.

Sur le plan pratique, la conception de jeux sérieux doit soit être capable de concilier les points de vue des professionnels du game design et des professionnels de l'enseignement (en tablant par exemple sur leurs compétences communes de joueurs), soit être menée par un individu ayant des compétences en didactique et en game design ${ }^{13}$.

Dans le cas contraire, les jeux sérieux continueront à être construits via les approches décrites au paragraphe 1.2, la sous-estimation de cette spécificité apparaissant comme une des causes principales des insuffisances constatées récemment qui ont conduit à remettre en cause la valeur ludique et pédagogique de l'offre actuelle en jeux vidéo éducatifs.

\section{Références}

Alvarez, J. (2007). Du jeu vidéo au serious game : approches culturelle, pragmatique et formelle. Thèse de doctorat en sciences de l'information et de la communication, sous la direction de Jean-Pierre Jessel et Gilles Methel, Université Toulouse II et III.

Brousseau, G. (1998). Théorie des situations didactiques. Grenoble : La Pensée sauvage.

Brousseau, G. (1990). Le contrat didactique : le milieu. Recherches en Didactique des Mathématiques. Grenoble : La Pensée sauvage.

Chen, J. (2006). Flow in games. Thèse. Disponible à : https://www.jenovachen.com/ flowingames/Flow_in_games_final.pdf.

Crawford, C. (2003). On game design, Indianapolis IN : New Riders.

Csíkszentmihályi, M. (2008). Flow: The Psychology of Optimal Experience, New York: Harper Collins Publishers.

El Mansouri, M. (2018). À table ! un serious game innovant sur l'alimentation : conception et ingénierie didactique. Nice : SEGAMED.

13 Rappelons que cette étude est issue d'un travail de thèse mené par M. El Mansouri sous la direction de N. Biagioli et R. Lozi qui articule les deux aspects théorique et pratique de la recherche sur la conception de jeux sérieux. M. El Mansouri a conçu un jeu sérieux pour enfants à partir du modèle sémiotique du gameplay pour apprendre en se fondant sur sa double expérience d'enseignante et de game designer (El Mansouri, 2018). Un prototype a été développé puis expérimenté dans différents contextes : scolaire, périscolaire, playtest (test en laboratoire sur un échantillon d'utilisateurs) et lors de festivals. Les premiers résultats sur un échantillon globalisé de 49 enfants en contexte scolaire et périscolaire ont montré que seuls $8 \%$ des enfants avaient identifié l'application numérique uniquement en tant qu'exercice et que $90 \%$ d'entre eux avaient le sentiment d'avoir appris des savoirs. 
El Mansouri, M. (2017a). Le jeu vidéo éducatif ou serious game, vecteur de savoir du $\mathrm{XXI}^{\mathrm{e}}$ siècle : processus de conception, ingénierie didactique et approche pédagogique. Conférence prononcée lors de la journée doctorale OMNSH 2. Penser et étudier les objets numériques, Montréal, 3 juin.

El Mansouri, M. (2017b). inédit, demi-journée doctorale, octobre.

El Mansouri, M. \& Biagioli N. (2016). Des jeux sérieux plus « sérieux » que ludiques? Nice : SEGAMED.

Fenouillet, F. (2014). Serious games : se former en s'amusant. Sciences humaines 257, 48-49.

Genvo, S. (2008). Caractériser l'expérience du jeu à son ère numérique : pour une étude du play design. Journée d'études "Les jeux vidéo : expériences et pratiques sociales multidimensionnelles ", Québec, Canada, 6 et 7 mai. Disponible à : http://www. ludologique.com/wordpress/?p=105.

Genvo, S. (2012). Penser les phénomènes de ludicisation à partir de Jacques Henriot, Sciences du jeu. 30 ans de Sciences du jeu à Villetaneuse. Hommage à Jacques Henriot [En ligne], 1 | 2013, mis en ligne le 01 octobre 2013. Disponible à : http:// journals.openedition.org/sdj/251. Doi : 10.4000/sdj.251.

Genvo, S. (2013). Penser la formation et les évolutions du jeu sur support numérique, Habilitation à diriger des Recherches. Mémoire soutenu à l'Université de Lorraine sous la direction de Jacques Walter. Disponible à : http://www.omnsh.org/sites/default/files/hdr_sg_t1.pdf.

Greimas, A. J. (1970). Du sens, Paris : Seuil.

Henriot J. (1989). Sous couleur de jouer, Paris : José Corti.

Koster, R. (2013). A Theory of fun for game design, Sebastopol CA : O 'Reilly.

Lavigne, M. (2015). Pour une approche critique des technologies numériques dans l'éducation, Colloque Ticemed 9 - Toulon, Avril.

Loquet, M. (2009). Jeu épistémique et jeu d'apprentissage dans les activités physiques, sportives et artistiques : vers une approche comparatiste en didactique. Note de synthèse pour l'Habilitation à Diriger des Recherches. Université de Rennes 2. Disponible à : http://www.aris-intervention-sport.org/sites/default/files/6/dim, $\% 20$ 11/12/2011\%20-\%2018:31/NoteSynthese\%20HDR\%20M.LOQUET_0.pdf.

Loup, G., Serna, A., George, S., Sanchez, E., Emin-Martinez, V., Bisognin, L. (2015). État de l'art et classification des JEN-livrable 2.1.1 du projet Jen.lab, avril 2015.

Perraudeau, M. (2006). Les stratégies d'apprentissage. Comment accompagner les élèves dans l'appropriation des savoirs. Paris : Armand Colin.

Schaeffer, J. M. (1999). Pourquoi la fiction?, Paris : Seuil.

Sensevy, G. (2011). Le sens du savoir. Éléments pour une théorie de l'action conjointe en didactique. Coll. Perspectives en éducation et formation. Bruxelles : De Boeck supérieur.

Sensevy, G. \& Mercier, A. (2007). Agir ensemble : l'action didactique conjointe du professeur et des élèves. Coll. Paideia. Rennes : Presses universitaires de Rennes.

Vygotski, L. (1997). Pensée et langage. Paris : La Dispute.

\section{(c) $(1) \Theta \Theta$}

«Attribution - Pas d'Utilisation Commerciale - Pas de Modification 4.0 International» (CC BY-NC-ND) 